\title{
EFEITO DO ÁCIDO INDOLBUTÍRICO E ÉPOCA DE COLETA NO ENRAIZAMENTO DE ESTACAS SEMI-LENHOSAS DO CACAUEIRO ${ }^{1}$
}

\author{
JOSÉ BASÍLIO VIEIRALEITE ${ }^{2} \&$ ANTONIO BALDO GERALDO MARTINS ${ }^{3}$
}

RESUMO: O objetivo do trabalho foi verificar o efeito do ácido indolbutírico (AIB) no enraizamento de estacas do cacaueiro. O delineamento experimental foi o inteiramente casualizado, em esquema fatorial 3 × 5 x 2, envolvendo 3 clones (Cepec 2008, CCN 51 e TSH 1188), 5 concentrações de ácido indolbutírico $\operatorname{AIB}\left(0 ; 1.000 ; 3.000 ; 6.000\right.$ e $\left.9.000 \mathrm{mg} \mathrm{kg}^{-1}\right)$, duas épocas do ano (verão e inverno), cinco repetições e 10 estacas por parcela. A avaliação do experimento foi realizada 120 dias após o plantio e analisadas as seguintes variáveis: percentagem de sobrevivência (SOB), número de brotações (NB), matéria seca das brotações (MSB), percentagem de estacas enraizadas (ENR), número de raízes (NR) e matéria seca de raízes (MSR). Os fatores época do ano e concentração de AIB apresentaram efeito significativo para as variáveis estudadas quando isolados e em interação com os clones. Os dados obtidos possibilitaram concluir que o valor médio da concentração ideal de AIB (CI) foi de $4.169 \mathrm{mg} \mathrm{kg}^{-1}$ e $3.985 \mathrm{mg} \mathrm{kg}^{-1}$ no verão e inverno, respectivamente. Verificou-se que os clones apresentam diferentes respostas em relação à CI de AIB e época do ano. De modo geral, as CIs no verão foram maiores que no inverno. Os resultados mostraram que a época de plantio e a concentração de AIB influenciaram de maneira mais expressiva na sobrevivência e enraizamento das estacas dos clones Cepec 2008 e CCN 51 e em menor intensidade no clone TSH 1188. Existe uma concentração ideal de AIB para cada um dos clones estudados.

Termos para indexação: Theobroma cacao L. estaquia, propagação, AIB.

\section{EFFECT OF INDOLBUTYRIC ACID AND CUTTING HARVESTING PERIOD ON ROOTING OF SEMI-HARDWOOD COCOA CUTTINGS}

\begin{abstract}
The objective of this work was to verify the effect of indolbutyric acid (IBA) on rooting of cocoa cuttings. The layout used was a complete randomized design in a 3 x 5 x 2 factorial scheme with three clones (Cepec 2008, CCN 51 e TSH 1188), five concentrations of indolbutyric acid - IBA - $\left(0,1.000,3.000,6.000\right.$ e $\left.9.000 \mathrm{mg} \mathrm{kg}^{-1}\right)$, two harvest periods (summer and winter), 10 cuttings per plot and five replications. At 120 days after planting, it was analyzed the following variables: survival percentage (SOB), shoot number (NB) shoots dry matter (MSB) and roots dry matter (MSR). The factors period of the year and IBA concentration showed significant effect in the variables studied isolated and in interaction with clones. The obtained data permit to conclude that the mean ideal concentrations (CI) of IBA were $4.169 \mathrm{mg} \mathrm{kg}^{-1}$ and $3.985 \mathrm{mg} \mathrm{kg}^{-1}$ in the summer and in the winter, respectively. It was verified that the clones showed different responses in regard to CI of IBA and year period. In general, the Cl's in the summer were higher than in the winter. The results showed that period of the year and IBA concentrations influenced expressively the survival and rooting of cuttings of Cepec 2008 and CCN 51 clones and, with lower intensity, the TSH 1188 clone. There is an ideal IBA concentration for each one of the studied clones.
\end{abstract}

Index Terms: Theobroma cacao L., rooting cuttings, propagation, IBA.

\section{INTRODUÇÃO}

A cacauicultura baiana foi estabelecida exclusivamente com mudas obtidas de sementes das variedades comuns e híbridas. Segundo Dias (2001), isso se configura como um dos raros casos entre espécies perenes cultivadas em que a reprodução seminal superou a propagação vegetativa.

O surgimento da vassoura-de-bruxa (Pereira et al., 1989), doença causada pelo fungo Crinipellis perniciosa (Stahel) Singer), provocou queda na produção e na produtividade da cacauicultura no Estado da Bahia (Sena-Gomes et al., 2000). Dentre as diferentes estratégias adotadas para o controle dessa doença, a seleção e a multiplicação de material botânico tolerante têm sido as mais utilizadas. A partir de 1997, a Comissão Executiva do Plano da Lavoura Cacaueira-Ceplac, disponibilizou os primeiros clones tolerantes para uso direto pelos agricultores. No entanto, devido à grande demanda pelo novo material, houve a necessidade de ajustar os métodos convencionais de propagação vegetativa do cacaueiro.

A propagação vegetativa de plantas permite a multiplicação

\footnotetext{
${ }^{1}$ (Trabalho 121-06). Recebido em 17-08-2006. Aceito para publicação em 15-06-2007. Parte da tese de doutorado do primeiro autor, apresentada à Universidade Estadual Paulista, Jaboticabal-SP.

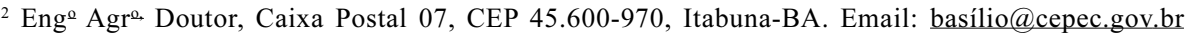

${ }^{3}$ Eng $^{\mathrm{o}}$ Agr ${ }^{\mathrm{o}}$, Professor Doutor do Departamento de Produção Vegetal, FCAV/UNESP, CEP 14.884-970, Jaboticabal-SP. Email: baldo@fcav.unesp.br
}

Rev. Bras. Frutic., Jaboticabal - SP, v. 29, n. 2, p. 204-208, Agosto 2007 
de progênies idênticas à planta a qual se obteve o material. Essa técnica tem sido largamente utilizada no mundo pela capacidade de utilização rápida dos ganhos genéticos obtidos nos programas de melhoramento (Haines, 1992; Hartmann et al., 1997) e por manter uma população de plantas uniforme (altura, taxa de crescimento, época de floração e colheita, e outras características fenotípicas) além de diminuir o tempo para início da produção (Hartmann et al., 1997).

Um dos principais desafios para os técnicos que trabalham com propagação vegetativa, é estabelecer as condições ideais para o bom enraizamento de cada espécie, por meio de protocolos ajustados que permitam a propagação de plantas em larga escala e com bons rendimentos (Hartmann et al., 1997).

Dentre os complexos aspectos que agem sobre o enraizamento de estacas, destacam-se o manejo das plantasmatrizes, relacionado com rejuvenescimento das plantas e as condições ambientais de água, temperatura, luz, carboidratos, relação $\mathrm{C} / \mathrm{N}$, anelamentos de caule, tipo de estaca e período de coleta (Martins, 1998).

Com relação às matrizes, é necessário observar o horário da coleta, tipo de armazenamento da estaca, auxinas, nutrição mineral e lixiviação de nutrientes. Quanto ao manejo das condições ambientais, são importantes as relações entre água, temperatura, fluxo de irradiância e qualidade do fotoperíodo, técnicas de aceleração de crescimento e fotossíntese da estaca (Hartmann et al., 1997).

Em se tratando do enraizamento de estacas de cacaueiro, as condições do viveiro podem influir no crescimento e afetar a capacidade de enraizamento (Pyke 1933; Cheesman, 1934 ;Evans,1953). Nesse contexto, Pyke (1933) destacou que a estaca semilenhosa é a que apresenta maior média de enraizamento, e que a presença de folhas nas estacas é necessária para o sucesso do enraizamento. A idade da planta-matriz também influencia no mesmo, sendo que plantas mais jovens o potencializam (Cheesman, 1934; Evans, 1953).

Evans (1953) relatou que as condições ótimas para o enraizamento de estacas de cacaueiro são de 90 a $100 \%$ de umidade relativa do ar, de 12 a $15 \%$ de efetiva luz do sol e temperaturas que não excedam $30^{\circ} \mathrm{C}$. Segundo esse autor, o meio de enraizamento deve ter apropriado suprimento de água e uma boa relação umidade do ar/água para a indução e formação de raízes.

Estudos realizados por Sena-Gomes et al. (2000) sobre concentrações de ácido indolbutírico (AIB) e tipos de estaca em clones tolerantes à vassoura-de-bruxa evidenciaram que o genótipo tem forte influência no enraizamento, havendo material com média superior a $70 \%$ e outros com taxas inferiores a $50 \%$. Por outro lado, Hartmann et al. (1997) afirmaram que não é viável economicamente a produção comercial em larga escala de material botânico com enraizamento abaixo de $50 \%$.

Estudos para ajustes ao protocolo de produção de mudas de cacaueiros na região cacaueira da Bahia são necessários, principalmente quando relacionados ao estádio fisiológico das estacas, clones, concentrações reguladoras de crescimento (AIB, AIA, ANA, etc.) e tamanho de tubetes. Adicionalmente, Faria et al. (2001) e Sacramento et al. (2001a) estudaram o enraizamento de estacas de cacaueiro em função do tempo, tamanho do tubete e tratamento com AIB, e concluíram que o início da emissão das raízes ocorreu entre 20 e 30 dias após o estaqueamento, e o crescimento da parte aérea, de 60 a 70 dias. Esses autores também verificaram que o tamanho do tubete mais adequado foi de 288 $\mathrm{cm}^{3}$ e que houve incremento nos índices de enraizamento e aumento significativo da matéria seca das raízes com a aplicação de AIB (Sacramento et al., 2001b).

O presente trabalho objetivou verificar o efeito do ácido indolbutírico (AIB) no enraizamento de estacas de cacaueiro coletadas em duas épocas do ano.

\section{MATERIAL E MÉTODOS}

O experimento foi conduzido nos viveiros do Instituto Biofábrica de Cacau (IBC), em Ilhéus, Bahia, no período de janeiro a outubro de 2003. Foram utilizados os clones provenientes do campo de produção de propágulos do IBC. Esses clones são os mais recomendados na região, por apresentarem boa produção e tolerância à vassoura-de-bruxa.

Para evitar desordens nutricionais, as plantas-matrizes receberam fertilização no solo conforme recomendação da Ceplac (Chepote et al., 2005). Os ramos utilizados para o preparo das estacas foram coletados pela manhã e imediatamente transportados para o galpão de estaqueamento, onde foram preparados e mantidos sob aspersão de água freqüente durante essa fase.

$\mathrm{O}$ delineamento experimental adotado foi o inteiramente casualizado, em esquema fatorial $3 \times 5 \times 2$, envolvendo 3 clones e 5 concentrações de AIB $\left(0 ; 1.000 ; 3.000 ; 6.000\right.$ e $\left.9.000 \mathrm{mg} \mathrm{kg}^{-1}\right)$, duas épocas do ano (verão e inverno) e cinco repetições, sendo cada parcela constituída por 10 estacas.

Foram utilizadas estacas semilenhosas oriundas de ramos plagiotrópicos com $20 \mathrm{~cm}$ de comprimento, com 3 a 4 gemas e 3 folhas reduzidas a dois terços de seu tamanho original. A seguir, as estacas foram tratadas com a mistura de AIB + talco e colocadas em tubetes plásticos com $288 \mathrm{~cm}^{3}$, contendo mistura do substrato comercial Plantmax ${ }^{\circledR}+$ fibra de coco + composto de tegumento de cacau na proporção 2:1:1 (v/v).

Após o estaqueamento, os tubetes foram colocados em bandejas de 54 células e transportados para viveiro com $50 \%$ de luminosidade, onde receberam nebulização intermitente de 30 segundos, a cada 4 minutos, onde permaneceram por um período de 60 dias. Nesse período, a temperatura média foi de $24^{\circ} \mathrm{C}$, e a umidade relativa do ar oscilou entre 80 e $90 \%$. Após esse período, os tubetes foram transferidos para outro viveiro com $70 \%$ de luminosidade e com a freqüência de nebulização reduzida, gradativamente, até alcançar 4 aspersões/dia, permanecendo por mais 60 dias.

A avaliação do experimento foi realizada 120 dias após o estaqueamento quanto às seguintes variáveis: sobrevivência (SOB), número de brotações (NB), matéria seca das brotações (MSB), estacas enraizadas (ENR), número de raízes (NR) e matéria seca de raízes (MSR).

Os dados obtidos foram submetidos à análise de variância, e as médias, comparadas pelo teste de Tuckey, a $5 \%$ de probabilidade. As interações significativas foram submetidas à análise de regressão, para o estudo do efeito do AIB. 


\section{RESULTADOS E DISCUSSÃO}

Na Tabela 1, são apresentados o resumo da análise de variância e os respectivos valores do Teste F para os parâmetros de enraizamento de estacas de cacaueiro. O fator clone isolado não apresentou significância para ENR, SOB e NB. Por outro lado, os fatores época de coleta e concentração de AIB apresentaram significância quando isolados e em interação, demonstrando a importância desses fatores e suas respectivas interações no enraizamento de estacas de cacaueiro. Os resultados encontrados para clone, isoladamente, são diferentes dos citados por Sena-Gomes et al. (2000). No entanto, os resultados do efeito época de coleta e concentração de AIB isolados ou em interação são corroborados por Cheesman (1934), Evans (1953) e Duarte (1954).

Estudos com outras importantes frutíferas têm demonstrado, também, que a época de coleta de estacas influencia na capacidade de seu enraizamento em várias espécies e mesmo entre cultivares ou clones da mesma espécie (Martins, 1998; Nachtigal et al., 1999; Scaloppi Junior \& Martins, 2003). Essas diferenças geralmente ocorrem devido à relação entre a época do ano e o estádio do ramo e da atividade fisiológica das plantas. Apesar do pequeno gradiente de variação climática da região sul da Bahia, no verão ocorre maior intensidade de refoliação das plantas, o que explica as diferenças obtidas no enraizamento, nesse período, comparado com o inverno (Tabela 2).

Para verificar o desdobramento da interação época x clone x concentração, foram ajustados modelos de regressão (Figuras 1 e 2). Os resultados mostram que as características avaliadas no verão, em todos os clones, independentemente da concentração de AIB, foram superiores àquelas encontradas no inverno, evidenciando a forte influência da época do ano para a coleta e estaqueamento do cacaueiro (Figuras 1 e 2).

Os resultados revelam que as concentrações em torno de $4.000 \mathrm{mg} \mathrm{kg}^{-1}$ promoveram as maiores percentagens de sobrevivência e enraizamento, número de raízes e de brotações, e massa seca de raiz e de brotação, enquanto em concentrações menores que $2.000 \mathrm{mg} \mathrm{kg}^{-1}$ e maiores que $6.000 \mathrm{mg} \mathrm{kg}^{-1}$, tendem a promover menores respostas dos carecteres avaliados, independentemente da época do ano (Figuras 1 e 2).

O padrão de comportamento dos fatores avaliados na regressão, para todas as variáveis estudadas, foi quadrático (Figuras 1 e 2). Os valores médios para os três clones, obtidos dos pontos de máxima das equações, mostraram que a variação da SOB foi de 75,5 e 50,7 \% ; o ENR 75,2 \% e 36,2 \%, o NR 20,4 e 11,1, o NB 6,9 e 2,7, a MSR 0,37 e 0,32 e a MSB foi 1,02 e 0,36 para o verão e o inverno, respectivamente (Tabela 2). Essas diferenças indicam que a época de coleta e de estaqueamento tem forte influência no enraizamento de estacas de cacaueiro.

Os dados obtidos possibilitaram calcular o valor da concentração ideal de AIB (CI) - ponto máximo da curva de regressão - que no verão foi de $4.169 \mathrm{mg} \mathrm{kg}^{-1}$, enquanto no inverno foi de $3.985 \mathrm{mg} \mathrm{kg}^{-1}$ (Tabela 2). Esses resultados indicam que o efeito da aplicação do AIB é dependente da fase fisiológica da planta como verificado por Hartmann et al. (1997) e Martins (1998) e da época do ano por Martins (1998).
Verificou-se que os clones mostram diferentes respostas em relação à CI de AIB e época do ano. De modo geral, as CIs, no verão, foram maiores do que as do inverno. Mudge (1988) relata que o efeito de auxinas na formação de raízes adventícias é altamente dependente da espécie, mas que, no entanto, prevalecem as condições ambientais e fisiológicas das plantas.

Pelos resultados das análises de regressão, pode-se verificar que o clone TSH 1188, à exceção do NR, não apresentou resposta significativa às concentrações de AIB para as características avaliadas no verão e no inverno. Esse resultado é discordante do encontrado por Faria et al. (2001), que verificaram valores superiores a $98 \%$ de enraizamento do clone TSH 1188 , com aplicação de $6.000 \mathrm{mg} \mathrm{kg}^{-1}$ de AIB.

O clone TSH 1188 apresenta média facilidade para enraizamento (Sena-Gomes et al., 2000), tendo esses autores encontrado valores inferiores a $55 \%$ e baixa resposta à concentração de AIB. Já os clones CCN 51 e Cepec 2008, que são caracterizados como de elevada e baixa capacidade de enraizamento, respectivamente (Del Campo \& Andia, 1997), nesse trabalho responderam à aplicação de $\mathrm{AIB}$, com aumentos no ENR, SOB, NR e NB (Figuras 1 A, C, E e 2 E).

A concentração de AIB utilizada no Instituto Biofábrica de Cacau, nos últimos anos, é, segundo Palácios (2000), de 6.000 $\mathrm{mg} \mathrm{kg}^{-1}$ para os clones atualmente propagados nessa empresa. Comparando-se esse valor com a CI, verifica-se que os valores para o clone Cepec 2008 e CCN 51 são 4.413 e $3.833 \mathrm{mg} \mathrm{kg}^{-1}$ e 4.096 e $4.523 \mathrm{mg} \mathrm{kg}^{-1}$ no verão e inverno, respectivamente. Em termos teóricos, essas concentrações permitiriam obter melhores resultados no cômputo geral das características avaliadas. Assim, os valores de CI estariam também indicando que o uso de uma mesma concentração para diferentes clones pode causar efeitos deletérios e aumentar os custos de produção devido ao consumo desnecessário de AIB.

TABELA 1 - Valores do teste de F obtidos para os fatores época, clones, concentração e respectivas interações em relação à sobrevivência (SOB), ao enraizamento (ENR), número de raízes (NR) e brotações (NB), e massa seca de raiz (MSR) e brotação (MSB). IlhéusBA. Instituto Biofábrica de Cacau, 2006.

\begin{tabular}{lcccccc}
\hline Causas de variação & $\mathrm{SOB}^{2}$ & $\mathrm{ENR}^{2}$ & $\mathrm{NR}^{3}$ & $\mathrm{NB}^{3}$ & $\mathrm{MSR}$ & $\mathrm{MSB}$ \\
\hline Época & $118,10^{* *}$ & $23,55^{* *}$ & $65,43^{* *}$ & $313,73^{* *}$ & $4,87^{* *}$ & $302,28^{* *}$ \\
Clone & $2,11^{\text {ns }}$ & $1,34^{\text {ns }}$ & $4,47^{*}$ & $2,91^{\text {ns }}$ & $10,58^{* *}$ & $8,07^{* *}$ \\
Concentração AIB & $14,57^{* *}$ & $10,84^{* *}$ & $45,22^{* *}$ & $9,79^{* *}$ & $5,94^{* *}$ & $2,87^{*}$ \\
Época x Clone & $24,44^{* *}$ & $41,91^{* *}$ & $58,25^{* *}$ & $16,69^{* *}$ & $0,27^{\text {ns }}$ & $63,3^{* *}$ \\
Época x Concentração & $3,44^{* *}$ & $1,95^{\text {ns }}$ & $14,86^{* *}$ & $3,32^{* *}$ & $3,39^{* *}$ & $2,10^{\text {ns }}$ \\
Clone x Concentração & $5,16^{* *}$ & $6,18^{* *}$ & $6,30^{* *}$ & $4,40^{* *}$ & $1,77^{\text {ns }}$ & $1,81^{\text {ns }}$ \\
Época x clone x concentração & $2,56^{* *}$ & $2,24^{*}$ & $4,21^{* *}$ & $2,72^{* *}$ & $0,91^{\text {ns }}$ & $3,18^{* *}$ \\
\hline CV (\%) & $\mathbf{4 2 , 4}$ & $\mathbf{3 5 , 5}$ & $\mathbf{2 2 , 0}$ & $\mathbf{3 1 , 0}$ & $\mathbf{5 3 , 2}$ & $\mathbf{6 2 , 9}$ \\
\hline
\end{tabular}

ns : não significativo; ${ }^{* *},{ }^{*}$ significância a $1 \%, 5 \%$ de probabilidade, respectivamente.

2 Dados transformados em arco-seno $\sqrt{x / 100} \mathrm{e}^{3}$ Dados transformados em $\sqrt{x+0,5}$. 
TABELA 2 - Índice de sobrevivência, enraizamento, número de raízes e de brotações, massa seca de raiz e brotação em estacas de cacaueiro, em função da concentração ideal de ácido indolbutírico ${ }^{1}$.

\begin{tabular}{|c|c|c|c|c|c|c|c|c|c|c|c|c|c|c|}
\hline \multirow[t]{2}{*}{ Época } & \multirow[t]{2}{*}{ Clone } & \multicolumn{2}{|c|}{$\begin{array}{c}\text { Sobrevivência } \\
(\%)\end{array}$} & \multicolumn{2}{|c|}{$\begin{array}{c}\text { Enraizamento } \\
(\%)\end{array}$} & \multicolumn{2}{|c|}{ Número de raízes } & \multicolumn{2}{|c|}{$\begin{array}{l}\text { Número de } \\
\text { Brotações }\end{array}$} & \multicolumn{2}{|c|}{ Massa seca de raiz } & \multicolumn{2}{|c|}{$\begin{array}{c}\text { Massa seca de } \\
\text { brotação }\end{array}$} & \multirow{2}{*}{$\begin{array}{c}\text { Média geral } \\
\mathrm{CI} \\
\left(\mathrm{mg} \mathrm{kg}^{-1}\right)\end{array}$} \\
\hline & & CI & VALOR & $\mathrm{CI}$ & VALOR & CI & VALOR & $\mathrm{CI}$ & VALOR & $\mathrm{CI}$ & VALOR & $\mathrm{CI}$ & VALOR & \\
\hline \multirow{5}{*}{$\frac{0}{\substack{d \\
\frac{1}{1}}}$} & CEPEC 2008 & 4.407 & 74.8 & 4.020 & 76.8 & 4.670 & 24.66 & 3.954 & 8.18 & 4.112 & 0.41 & 3.852 & 0.31 & 4.413 \\
\hline & TSH 1188 & 4.124 & 65.8 & 4.018 & 65.7 & 4.234 & 16.1 & 2.812 & 6.37 & 4.869 & 0.4 & 4.750 & 1.02 & 3.999 \\
\hline & CCN 51 & 4.479 & 85.8 & 4.098 & 93.3 & 4.480 & 20.35 & 3.623 & 6.33 & 4.612 & 0.3 & 3.283 & 0.73 & 4.096 \\
\hline & Média & 4.337 & 75.47 & 4.045 & 75.2 & 4.461 & 20.37 & 3.463 & 6.96 & 4.531 & 0.37 & 3.962 & 1.02 & 4.169 \\
\hline & esvio-padrão & 187.7 & 10.02 & 45.6 & 13.89 & 218.6 & 4.28 & 587.6 & 1.06 & 384.9 & 0.06 & 739.6 & 0.36 & 216.52 \\
\hline \multirow{3}{*}{ 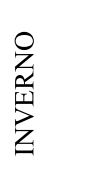 } & CEPEC 2008 & 3.978 & 50 & 3.800 & 30 & 3.675 & 8.8 & 3.218 & 3.01 & 4.931 & 0.37 & 3.763 & 0.34 & 3.833 \\
\hline & TSH 1188 & 4.413 & 47.5 & 3.084 & 33.7 & $\mathrm{~ns}$ & $\mathrm{~ns}$ & 3.355 & 3.08 & 4.056 & 0.32 & 3.085 & 0.55 & 3.599 \\
\hline & CCN 51 & 3.768 & 54.7 & 4.321 & 45 & 4.928 & 13.4 & 3.130 & 2.17 & 4.415 & 0.27 & 4.962 & 0.18 & 4.523 \\
\hline & Média & 4.053 & 50.7 & 3.735 & 36.22 & 4.302 & 11.1 & 3.234 & 2.75 & 4.467 & 0.32 & 3.937 & 0.36 & 3.985 \\
\hline & esvio-padrão & 329 & 3.66 & 621.1 & 7.81 & 886.8 & 3.25 & 113.4 & 0.51 & 439.8 & 0.05 & 950.5 & 0.19 & 480.39 \\
\hline & Média geral & 4.195 & 63.09 & 3.890 & 55.71 & 4.397 & 15.74 & 3.349 & 4.86 & 4.499 & 0.35 & 3.949 & 0.69 & 4.077 \\
\hline
\end{tabular}

Legenda: CI - Concentração ideal. ${ }^{1}$ Valores obtidos dos pontos ótimos das equações da análise de regressão, com significância de 5 \%
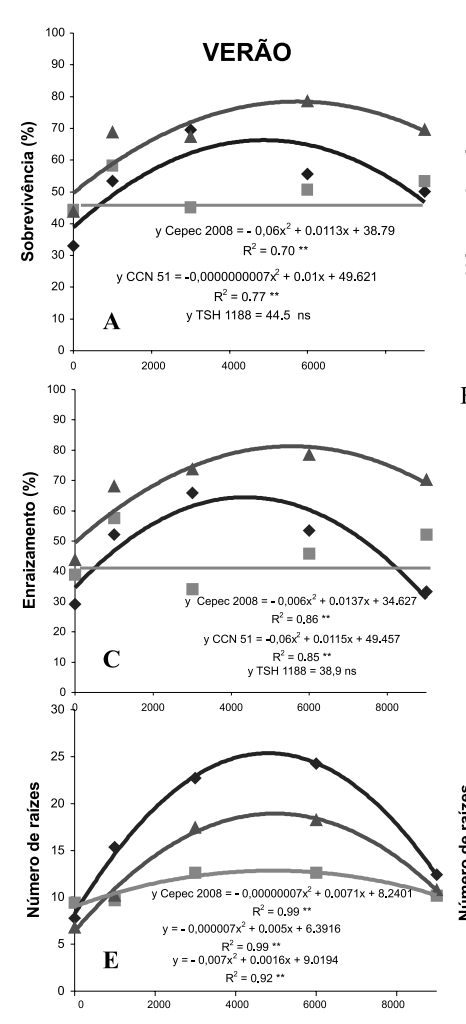

AlB $\left(\mathbf{m g ~ k g}^{-1}\right)$

CEPEC 2008
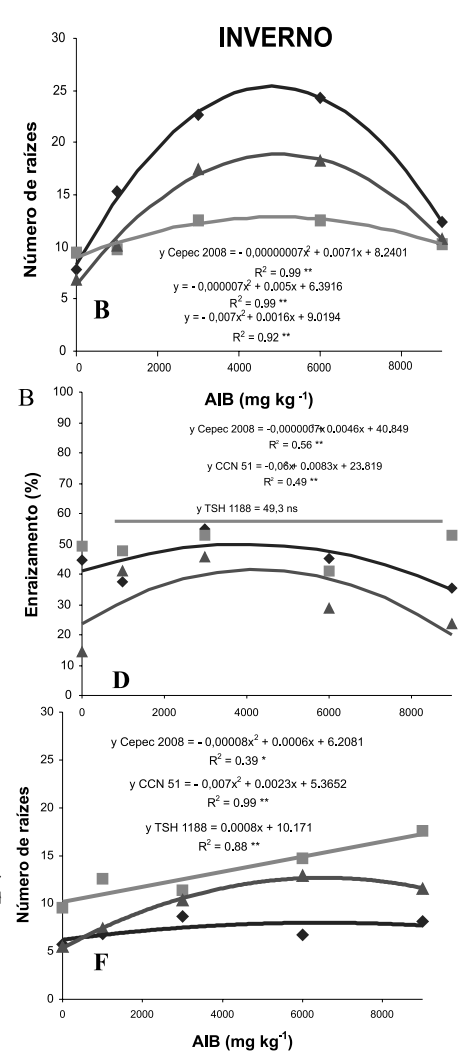

CCN 51

FIGURA 1 - Efeito de diferentes concentrações de AIB na percentagem de sobrevivência de estacas de três clones de cacaueiro, no verão (A) e no inverno (B); na percentagem de estacas enraizadas no verão (C) e no inverno (D), e no número de raiz no verão $(\mathrm{E})$ e no inverno $(\mathrm{F})$.
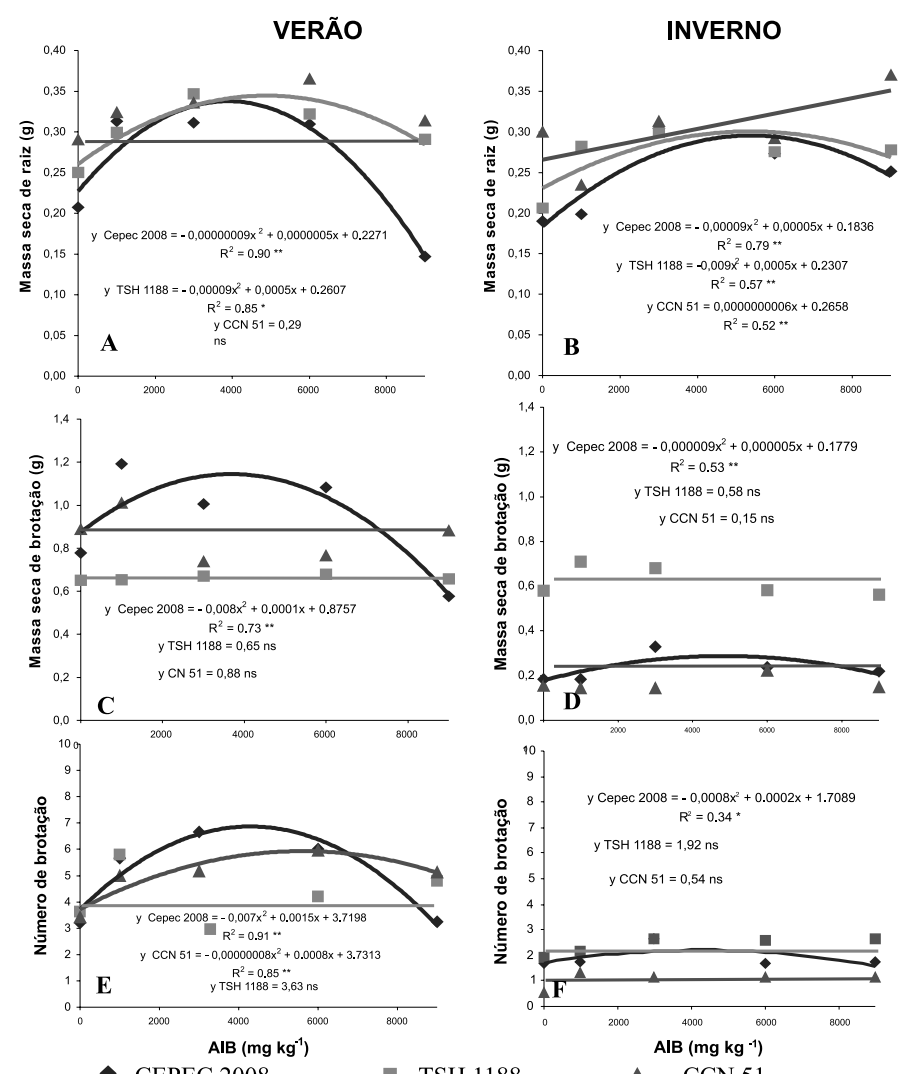

- CEPEC 2008

- TSH 1188

- CCN 51

FIGURA 2 - Efeito de diferentes concentrações de AIB na massa seca de raiz de três clones de cacaueiro, no verão (A) e no inverno (B); na massa seca de brotação no verão (C) e no inverno (D), e no número de brotação no verão (E) e no inverno (F). 


\section{CONCLUSÃO}

Estacas colhidas no verão respondem melhor ao enraizamento que estacas colhidas no inverno, e clones de cacaueiro respondem diferentemente às concentrações de AIB, existindo uma concentração ideal deste regulador de crescimento para cada clone estudado.

\section{REFERÊNCIAS}

CHEESMAN, E. E. The vegetative propagation of cacao. Empire Journal of Experimental Agriculture, Oxford, v.2. n.5, p. 40-50, 1934.

CHEPOTE, R. E; SODRÉ, G. A.; REIS, E. L.; PACHECO, R. G; MARROCOS, P. C. L.; SERÔDIO, M. H. de C. F.; VALLE, R. $R$. Recomendações de corretivos e fertilizantes na cultura do cacaueiro no sul da Bahia: $2^{\mathrm{a}}$ aproximação. Ilhéus: Ceplac/ Cepec, 2005. $36 \mathrm{p}$.

DEL CAMPO, E. C.; ANDIA, F. C. Cultivo y benefício del cacao CCN 51. Quito: Ed. El Conejo, 1997. 136 p

DIAS, L.A. S. Melhoramento genético do cacaueiro. ViçosaMG: FUNAPE-UFG, 2001.501 p.

DUARTE, O. Mejores preparaciones hormonales para el enraizamiento de las estacas de cacao. In: REUNION COMITÉ TÉCNICO INTERAMERICANO DE CACAO, 5. 1954, Turrialba. $13 \mathrm{p}$.

EVANS, H. Investigations on the propagation of cacao. Tropical Agriculture, St. Augustine, v.28, p.147-203, 1953.

FARIA, J. C.; SACRAMENTO, C. K.; PALACIOS, J. B.; CERQUEIRA, L. S. Enraizamento e crescimento de estacas herbáceas do cacaueiro (clones Cepec 42, TSH 516 e TSH 1188) em função da aplicação do ácido indolbutírico (AIB). In: CONGRESSO BRASILEIRO DEFISIOLOGIA VEGETAL, 8., 2001. Ilhéus. Anais... Ilhéus: Ceolac, 2001. 1 CD-ROM

HAINES, R. J. Mass propagation by cuttings, biotechnologies and capture of genetic gain. In: SYMPOSIUM IN IUFR'S CENTENNIAL YEAR: mass production technology for genetically improved fast growing forest tree species, 1992, Bordeaux. Syntheses... Paris: Afocel/Iufro, 1992. p.128-144.

HARTMANN, H.T.; KERSTER, D.E.; DAVIES JR., F.T.; GENEVE, R.L. Plant propagation: principles and pratices. 6.ed. New Jersey: Prentice-Hall, 1997. p.276-501.

MARTINS, A.B.G. Enraizamento de estacas enfolhadas de três variedades de lichia Litchi chinensis Sonn. 1998. 100f. Tese (Doutorado em Produção Vegetal) - Universidade Estadual Paulista, Faculdade de Ciências Agrárias e Veterinárias, Jaboticabal-SP, 1998.

MUDGE, K.W. Effect of ethylene on Rooting. In: DAVIS, T.D.; HAISSIG, B.E.; SANKHLA, N. (Eds). Adventitions root formation in cuttings. Portland : Dioscoredes Press, 1988. p.150-161.

NACHTIGAL, J. C; PEREIRA, F. M.; CAMPO DALL'ORTO, F. A.; OJIMA, M.; MARTINS, F. P. Propagação vegetativa do umezeiro (Prumus mume) por meio de estacas herbáceas. Revista Brasileira de Fruticultura, Jaboticabal, v. 21, n. 2, p. 226-228, 1999.
PALÁCIOS, J.B. Disponibilidade de material de clonagem no campo: logística, operação/produção e custos. In: CONGRESSO ATUALIZAÇÃO SOBRE PRODUÇÃO MASSAL DE PROPÁGULOS DE CACAU GENETICAMENTE MELHORADO, 1998, Ilhéus. Atas... Ilhéus: CEPEC, 1998. p.98-103.

PEREIRA, J. L.; RAM, A.; FIGUEIREDO, J. M. de e ALMEIDA, L. C. C. Primeira ocorrência de vassoura-de-buxa na principal região produtora de cacau do Brasil. Ilhéus-BA, Ceplac/ Cepec, Revista Agrotrópica, Ilhéus, v. 1, n.1, p. 79-81 1989.

PYKE, E. E. The vegetative propagation of cacao. II. Softwood cuttings. Annual Report on Cacao Research, Trinidad, v.2, p.3-9, 1933.

SACRAMENTO, C.K.; FARIA, J.C.; PALACIOS, J.B.; CERQUEIRA, L.S. Enraizamento e crescimento de estacas de cacaueiro (clone TSH 1188) em função do tempo de estaqueamento. In: CONGRESSO BRASILEIRO DE FISIOLOGIA VEGETAL, 8., 2001. Ilhéus-BA. Anais... Ilhéus: CEolac, 2001a. 1 CD-ROM

SACRAMENTO, C. K.; FARIA, J. C.; PALACIOS, J. B. Enraizamento e crescimento de estacas herbáceas de cacaueiro (clone TSH 516 e CEPEC 42) em função do tamanho do tubete IN: CONGRESSO BRASILEIRO DE FISIOLOGIA VEGETAL, 8., 2001, Ilhéus. Anais... Ilhéus: CEolac,, 2001b. 1 CD-ROM

SCALOPPIJUNIOR, E.J.; MARTINS, A.B.G. Clonagem de quatro espécies de Annonaceae potenciais como porta-enxertos. Revista Brasileira de Fruticultura, Jaboticabal, v. 25, n. 2, p. 283-289, 2003

SENA-GOMES, A.R.; CASTRO, G.C.; MORENO-RUIZ, M.M.; ALMEIDA, H.A. Avanços na propagação clonal do cacaueiro no sudeste da Bahia. In: PEREIRA, J. L. et al. (Eds). In: CONGRESSO ATUALIZAÇÃO SOBRE PRODUÇÃO MASSAL DE PROPÁGULOS DE CACAU GENETICAMENTE MELHORADO, 1998, Ilhéus. Atas... Ilhéus: CEolac ,2000. p.85-89. 